\title{
2,6-Dicarboxypyridinum chlorochromate: an efficient oxidizing agent for the very fast oxidation of Hantzsch 1,4- dihydropyridines
}

\author{
Majid M. Heravi, Khadijeh Bakhtiari, and Hossein A. Oskooie \\ Department of Chemistry, School of Sciences, Azzahra University, Vanak, Tehran, Iran \\ E-mail: mmh1331@yahoo.com
}

\begin{abstract}
2,6-Dicarboxypyridinum chlorochromate (2,6-DCPCC) was used as a highly effective oxidizing agent for the very fast oxidation of dihydropyridines to their corresponding pyridine derivatives under simple and relatively mild conditions in excellent yields.
\end{abstract}

Keywords: Hantzsch 1,4-dihydropyridines, 2,6-dicarboxypyridinum chlorochromate, 2,6DCPCC, pyridine derivatives, oxidation

\section{Introduction}

Hantzsch 1,4-dihydropyridines (Hantzsch1,4-DHP) have been extensively utilized as the analogs of $\mathrm{NAD}(\mathrm{P}) \mathrm{H}$ coenzymes to study the mechanism and the synthetic potential of various redox processes. ${ }^{1,2}$ In addition, Hantzsch 1,4-dihydropyridines based drugs such as Nifedipine and Niguldipine are widely used as calcium channel blockers for the treatment of cardiovascular disorder including angina, hypertension and cardiac arrhythmias. ${ }^{3}$ Both during the redox processes $^{2}$ and in the course of drug metabolism, ${ }^{4} 1,4$-DHP systems are oxidatively transformed into the corresponding pyridine derivatives. Consequently, this aromatization reaction continues to attract the attention of researchers for the discovery of milder and general protocols applicable to a wide range of 1, 4-dihydropyridines. A number of methods and reagents have been reported recently in the literature for this purpose. ${ }^{5-15}$

Some of these methods suffer from disadvantages such as the use of strong or toxic oxidants, the requirement of severe conditions or need excess of the oxidants. Other drawbacks are long reaction times, production of by products, the lower yields of products and/or the requirement of tedious work-up procedures.

In continuation of our program to develop new methods for oxidation of Hantzsch 1,4DHPs, ${ }^{16-19}$ herein, we wish to report a very convenient, clean and efficient approach for the oxidation of Hantzsch 1,4-dihydropyridines using 2,6-dicarboxypyridinium chlorochromate (2,6DCPCC) (Scheme 1). This reagent has been used as an oxidant for a variety of substrates. ${ }^{20-22}$ 


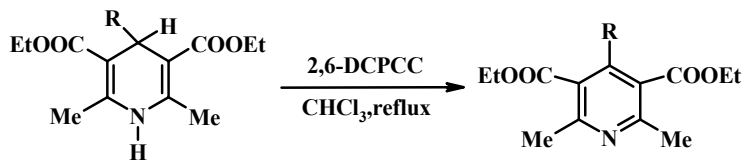

\section{Scheme 1}

\section{Results and Discussion}

In fact, refluxing a mixture of 1,4-DHP( $1 \mathrm{mmol})$, and 2,6 -DCPCC $(0.5 \mathrm{mmol})$ in chloroform gave in very short time and after simple filtration, desired pyridine derivatives in excellent yields. The salient features of this reaction are the stability of the substituents at 4-position confirmed by spectroscopic and physical data and very short reaction times. The reactions take place almost immediately.

In order to show the advantages of this reagent over some other oxidants, we have compared our results with those reported in Table 2. The results show that 2,6-DCPCC promotes the reaction more effectively than other reagents. Reaction in the presence of those reagents which are listed in Table 2 required longer reaction times.

Table 1. Oxidation of 1, 4-DHPs using 2,6-DCPCC

\begin{tabular}{|c|c|c|c|c|c|}
\hline \multirow[t]{2}{*}{ Entry } & \multirow[t]{2}{*}{$\mathrm{R}$} & \multirow[t]{2}{*}{ Yield $(\%)^{\mathrm{a}}$} & \multirow[t]{2}{*}{ time (min) } & \multicolumn{2}{|c|}{ m.p $\left({ }^{\circ} \mathrm{C}\right)^{\mathrm{b}}$} \\
\hline & & & & Observed & Reported \\
\hline 1 & $\mathrm{H}-$ & 90 & 2 & 70 & $69-70^{[24]}$ \\
\hline 2 & Et- & 88 & 6 & oil & $\mathrm{oil}^{[5]}$ \\
\hline 3 & & 90 & 5 & 61 & $61-62^{[5]}$ \\
\hline 4 & & 89 & 3 & $66-67$ & $66-67^{[24]}$ \\
\hline 5 & & 87 & 10 & $62-63$ & $61-63^{[24]}$ \\
\hline 6 & & 92 & 2 & 50 & $50^{[25]}$ \\
\hline 7 & & 91 & 7 & oil & oil $^{[24]}$ \\
\hline
\end{tabular}

a) Yields refer to the isolated products.

b) Products exhibited physical properties in accordance with the assigned structures. 
Table 2. Comparison of 2.6-DCPCC with some the other reagents for oxidation of Hantzsch 1,4DHPs

\begin{tabular}{cccc}
\hline Reagent & Conditions & Time $(\mathrm{h}) /$ Yield $(\%)$ & Reference \\
\hline Solid supported PCC & $\mathrm{CH}_{2} \mathrm{Cl}_{2}, \mathrm{rt}$ & $1-24 \mathrm{~h} / 90$ & 10 \\
PDC & $\mathrm{DMF}$ & $1 \mathrm{~h} / 77-89$ & 27 \\
$\mathrm{Bi}\left(\mathrm{NO}_{3}\right)_{3} .5 \mathrm{H}_{2} \mathrm{O}$ & $\mathrm{ACOH}, \mathrm{rt}$ & $1-14 / 50-90$ & 12 \\
$\mathrm{DDQ}$ & $\mathrm{CHCl}_{3}, 20^{\circ} \mathrm{C}$ & $1 / 73-90$ & 24 \\
$\mathrm{TPCD}$ & $\mathrm{ACOH}, \mathrm{rt}$ & $3-6 / 70-87$ & 13 \\
$t \mathrm{BuOOH}$ & $100^{\circ} \mathrm{C}$ & $0.75-6 / 74-87$ & 26 \\
$\mathrm{RuCl}_{3} / \mathrm{O}_{2}$ & $\mathrm{AcOH}_{2}, \mathrm{rt}$ & $12-100 / 20-75$ & 25 \\
$2.6-\mathrm{DCPCC}$ & $\mathrm{CHCl}_{3}, \mathrm{reflux}$ & $2-10(\mathrm{~min}) / 87-91$ & This work \\
\hline
\end{tabular}

\section{Conclusions}

In conclusion, a readily prepared reagent can be used as a highly effective rapid, mild and inexpensive oxidant for aromatization of Hantzsch 1,4-DHPs to pyridine derivatives. The advantages of this reaction are very short reaction times, very easy and clean work-up and excellent yields. In terms of reaction time, to our best of knowledge, this reagent seems to be one of the fastest oxidant for the 1, 4-DHPs among known oxidants.

\section{Experimental Section}

General Procedures. All the dihydropyridines were prepared according to the literature procedure, using the appropriate aldehydes, ammonia and ethyl acetoacetate. ${ }^{23}{ }^{1} \mathrm{H}$ NMR spectra were recorded on a Bruker AQS AVANCE-300MHz spectrometer using TMS as an internal standard ( $\mathrm{CDCl}_{3}$ solution). IR spectra were recorded from $\mathrm{KBr}$ disk on the FT-IR Bruker Tensor 27. All products were known; their physical and spectroscopic data were compared with those of authentic samples and found to be identical.

Preparation of 2,6-DCPCC ${ }^{20}$. To a solution of chromium trioxide $(0.1 \mathrm{~mol}, 10 \mathrm{~g})$ in hydrochloric acid $(0.11 \mathrm{~mol}, 18 \mathrm{~mL})$, pyridine- 2,6-dicarboxylic acid $(0.1 \mathrm{~mol}, 16.7 \mathrm{~g})$ was added over $5 \mathrm{~min}$ at $-5^{\circ} \mathrm{C}$. The resulting solution was stirred at $-5^{\circ} \mathrm{C}$ for $2 \mathrm{~h}$ by which time a yelloworange solid precipitated. The crystals were collected on a sintered glass funnel and dried in vacuum, yield $24.5 \mathrm{~g}, 90 \%$ (Cr\% calcd $17.13 \%$, found $17.03 \%$ ).

\section{Oxidation of Hantzsch 1,4-DHPs using 2,6-DCPCC: General procedure}

To a chloroform solution $(5 \mathrm{~mL})$ of 2,6-DCPCC $(0.5 \mathrm{mmol})$, Hantzsch 1,4-DHP (1mmol) was added. The reaction mixture was refluxed for the specified time. The progress of reaction was 
monitored by TLC using petroleum ether: Ethyl acetate as eluent. Upon completion of the reaction, the mixture was filtered on silica gel pad. The filtrate was evaporated to dryness under reduced pressure to afford the pure product. The results are summarized in Table 1.

\section{Spectral and physical data for selected compounds:}

1. Yield: $90 \%$; mp: $70^{0} \mathrm{C}$; (lit. $\left.{ }^{24} 69-70\right)$; IR $\bar{v}$ (KBr): 755, 1553, 1600, 1730, 2923, $2965 \mathrm{~cm}^{-1} ;{ }^{1} \mathrm{H}$ NMR (300 MHz, $\left.\mathrm{CDCl}_{3}\right): \delta=1.42(\mathrm{t}, 6 \mathrm{H}, \mathrm{J}=7.2 \mathrm{~Hz}), 2.85(\mathrm{~s}, 6 \mathrm{H}), 4.40(\mathrm{q}, 4 \mathrm{H}, \mathrm{J}=7.2 \mathrm{~Hz}), 8.68$ (s, $1 \mathrm{H})$.

4. Yield: 89\%, mp: 66-670 C; (lit. $\left.{ }^{24} 66-67\right)$; IR $\bar{v}(\mathrm{KBr}): 2976,1730,1561,1238,1107 \mathrm{~cm}^{-1} ;{ }^{1} \mathrm{H}$ NMR (300 MHz, $\left.\mathrm{CDCl}_{3}\right): \delta=0.90(\mathrm{t}, 6 \mathrm{H}, \mathrm{J}=7.2 \mathrm{~Hz}), 2.60(\mathrm{~s}, 6 \mathrm{H}), 4.00$ (q, 4H, J=7.2 Hz), 7.2-7.4 $(\mathrm{m}, 5 \mathrm{H})$.

7. Yield: $91 \%$, oil; IR $\bar{v}(\mathrm{KBr}): 1046,1107,1561,1575,1730,2984,3075 \mathrm{~cm}^{-1} ;{ }^{1} \mathrm{H}$ NMR $(300$ $\mathrm{MHz}_{\mathrm{CDCl}}$ ): $\delta=1.12(\mathrm{t}, 6 \mathrm{H}, \mathrm{J}=6.9 \mathrm{~Hz}), 2.49(\mathrm{~s}, 6 \mathrm{H}), 4.18(\mathrm{q}, 4 \mathrm{H}, \mathrm{J}=6.9 \mathrm{~Hz}), 6.39$ (d, 1H, J=3.3 $\mathrm{Hz}), 6.54$ (d, 1H, J=3.3 Hz), 7.42 (br s, 1H).

\section{References}

1. Stout, D. M.; Meyers, A. I. Chem. Rev. 1982, 82, 223.

2. Kill, R. J.; Widdowson, D. A. In Bioorganic Chemistry, Van Tamelen, E. E.; Ed.; Academic Press: New York, 1978; p 239.

3. Triggle, D. J. In Comprehensive Medicinal Chemistry; Emmett, J. C.; Vol. Ed., Pergamon: Oxford, 1990; Vol. 3, Ch. 14.1.

4. Janis, R. A.; Triggle, D. J. J. Med. Chem. 1983, 25, 775.

5. Nasr-Esfahani, M.; Moghadam, M.; Tangestaninejad, Sh.; Mirkhani, V.; Momeni, A. R. Bioorg. Med. Chem. Lett. 2006, 14, 2720.

6. Moghadam, M.; Nasr-Esfahani, M.; Tangestaninejad, Sh.; Mirkhani, V. Bioorg. Med. Chem. Lett. 2006, 16, 2026.

7. Balogh, M.; Hermecz, I.; Meszaros, Z.; Laszlo, P. Helv. Chim. Acta. 1984, 67, 2270.

8. Eynde, J. J. V.; Orazio, R. D.; Haverabeke, Y. V. Tetrahedron 1994, 50, 2479.

9. Grinsteins, E.; Stankevice, B.; Duburs, G. Kim. Geterotsikl. Soedin. 1967, 1118; Chem. Abstr. 69, 77095.

10. Eynde, J. J. V.; Mayence, A.; Maquestiau, A. Tetrrahedron 1992, 48,463.

11. Delgado, F.; Alvarez, C.; Garcia, O.; Penieres, G.; Marques, C. Synth. Commun. 1991, 21, 2137.

12. Mashraqui, S. H.; Karnik, M. A. Synthesis 1998, 713.

13. Wang, B.; Hu, Y. Synth. Commun. 1999, 29, 4193.

14. Maquestiau, A.; Mayence, A.; Eynde, J. J. V. Tetrahedron Lett. 1991, 32, 3839.

15. Khadikar, B.; Borkat, S. Synth. Commun. 1998, 28, 207. 
16. (a) Heravi, M. M.; Bakhtiari, Kh.; Oskooie, H. A.; Hekmatshoar R. Heterocyclic Commun. 2006, 12, 209. (b) Heravi, M. M.; Behbahani, F. K.; Oskooie, H. A.; Hekmatshoar, R. Tetrahedron Lett. 2005, 46, 2775.

17. Heravi, M. M.; Ghassemzadeh, M.; Phosphorus Sulfur and Silicon 2005, 180, 347.

18. Heravi, M. M.; Dirkwand, F.; Oskooie, H. A.; Ghassemzadeh, M. Heterocyclic Commun. 2005, 11,75 .

19. (a) Heravi, M. M.; Moosavi, F. S. S.; Beheshtiha, Y. S.; Ghassemzadeh, M. Heterocyclic Commun. 2004, 10, 415. (b) Heravi, M. M.; Ghassemzadeh, M. Heterocyclic Commun. 2004, 10, 465. (c)Tajbakhsh, M.; Heravi, M. M.; Hosseini, A.; Shahrezaiee, A. Phosphorus Sulfur and Silicon 2003, 178, 773. (d) Heravi, M. M.; Derikvand, F.; Oskooie, H. A.; Hekmatshoar, R. J. Chem. Res. 2006, 168. (e) Heravi, M. M.; Derikvand, F.; Oskooie, H. A.; Hekmatshoar, R. Synth. Commun. 2006, 36, 77. (f) Fotouhi, L.; Khaleghi, S.; Heravi, M. M. Lett. Org. Chem. 2006, 3, 111.

20. Hosseinzadeh, R.; Tajbakhsh, M.; Niaki, M. Y. Tetrahedron Lett. 2002, 43, 9413.

21. Tajbakhsh, M.; Hosseinzadeh, R.; Niaki, M. Y. J. Chem. Res. 2002, 508.

22. Tajbakhsh, M.; Hosseinzadeh, R.; Shakoori, A. Tetrahedron Lett. 2004, 45, 1889.

23. Hantzsch, A. Condensationprodukte aus Aldehydammoniak und Ketoniartigen Verbindungen. Ber. 1881, 14,1637.

24. Eynde, J. J. V.; Delfosse, F.; Mayence, A.; Haverbeke, Y. V. Tetrahedron 1995, 51, 6511.

25. Mashraqui, S. H.; Karnik, M. A. Tetrahedron Lett. 1998, 39, 4895.

26. Chavan, S. P.; Dantal, S. W.; Kalkote, U. R.; Jyothirmai, V. S.; Kharul, R. K. Synth. Commun. 1998, 28, 2789.

27. Ko, K. -Y.; Park, J. Y. Bull. Korean Chem. Soc. 1995, 16, 200. 